EDPSWEB/webdav/site/mySite/shared/Documents/EDPS/Publications/ Annualreport/2013/AR2013_summary_DE.pdf

\section{Projekt DIN-Norm zu Datenschutz-Löschfristen}

Im Dezember 2012 stellte Secorvo die DIN-INS-Leitlinie zur „Entwicklung eines Löschkonzepts mit Ableitung von Löschfristen für personenbezogene Daten "vor. Die Leitlinie unterstützt verantwortliche Stellen dabei, ihre rechtlichen Pflichten zur Löschung personenbezogener Daten zu erfüllen. Sie gibt Empfehlungen für die Inhalte und den Aufbau eines Löschkonzepts sowie die Zuordnung der Verantwortlichkeiten. Der Schwerpunkt liegt auf einer effizienten Vorgehensweise zur Entwicklung von Löschregeln für Datenarten unter Verwendung von vereinheitlichten Löschklassen.

Die Leitlinie bietet die Chance, durch eine einheitliche Vorgehensweise branchenweite oder sogar branchenübergreifende Löschregeln für personenbezogene Daten festzulegen. Diese würden es Unternehmen erleichtern, angemessene Löschprozesse zu etablieren. Ende 2013 startete daher ein von den Unternehmen Blancco, DATEV, Deutsche Bahn, Secorvo und Toll Collect gefördertes Projekt, in dem die Leitlinie derzeit zu einer DIN-Norm weiterentwickelt wird. Am 12.02.2014 beschloss der DIN-Arbeitskreis für Identitätsmanagement und Datenschutz-Technologien (AK 05 im NIA 27) die Aufnahme eines entsprechenden Normungsprojekts. Die Norm für Löschkonzepte soll spätestens im Herbst 2015 verabschiedet werden.

Die veröffentlichte Leitlinie gibt bereits heute wesentliche Hilfestellung für die Entwicklung eigener Löschkonzepte. Sie ist zum Download verfügbar unter http://www.secorvo.de/publikationen/din-leitlinie-loeschkonzepthammer-schuler-2012.pdf

\section{Acronis Studie: Das IT-Sicherheitsbewusstsein der Deutschen}

Eine am 27.03.2014 veröffentlichte repräsentative Studie von der GfK im Auftrag von Acronis hat das Klischee vom sicherheitsbewussten Deutschen bestätigt: demnach sichern 89,3\% der befragten Deutschen ihre Daten mit einem Backup. Nur bei etwa $10 \%$ sind die Daten ungesichert. In Sachen Datensicherungsverhalten zeichnen sich deutliche Unterschiede $a b$, insbesondere zwischen den Generationen.

An der Acronis Backup Studie beteiligten sich insgesamt 1.095 Männer und Frauen ab 14 Jahren.

Hier die wichtigsten Ergebnisse im Überblick:

Etwa 10\% sichern ihre Daten nicht - Häufigkeit variiert Die Zahl der Personen, die kein Backup durchführen, liegt immer noch bei gut $10 \%$. Vor allem Frauen führen seltener eine Sicherung ihrer Daten durch. Bei der Generation 60+, 1 Personen- Haushalten sowie Arbeitslosen ist die Bereitschaft auf eine Datensicherung zu verzichten am Höchsten. Im Gegensatz dazu stehen Beamte und Selbstständige mit 97,1\% bzw. 94,1\%. Die Häufigkeit eines Backups variiert allerdings stark. $44,5 \%$ sichern nur nach Bedarf, $25 \%$ zumindest wöchentlich oder öfter. Dabei sichern die Deutschen hauptsächlich spezielle Laufwerke/Ordner/Dokumente (File Backup) statt des gesamten PCs einschließlich Betriebssystem mit allen Daten, Applikationen und Einstellungen (Image Backup). Im Not- fall bedeutet das allerdings, dass das Betriebssystem (inklusive Updates) ebenso wie alle Einstellungen neu installiert werden müssen.

\section{Verhaltene Nutzung der Cloud}

Studien haben bereits gezeigt, dass die externe Festplatte ein beliebter Speicherort für elektronische Daten ist. In einer repräsentativen Bitkom-Studie zum Thema Datenschutz im Internet belegt die externe Festplatte Rang 3 der externen Speichergeräte. Auch die Ergebnisse der Acronis Studie bestätigen die Beliebtheit der externen Festplatte als Speicherort. 56,4\% nutzen sie für die Ablage von gesicherten Daten, der Anteil an NAS (8,3\%) ist dagegen eher gering. Die Nutzung der Cloud ist insgesamt sehr verhalten, in der Tendenz jedoch steigend. Das liegt auch daran, dass 20\% der jungen Deutschen die Cloud bereits nutzen, bei den über 60-Jährigen sind dies nur 5,5\%.

Mehr Aufmerksamkeit für wichtige Daten bei Jüngeren Außerdem wollte Acronis wissen, wie die Deutschen mit ihren wichtigsten Daten umgehen. Dabei stellte sich heraus, dass nur $50 \%$ ihren wichtigen Daten einen besonderen Stellenwert einräumen. Während 59\% der Personen im Alter ab 60 Jahren alle Daten im regulären Backup abdecken, schenkt die junge Generation im Alter bis 29 Jahre mit 66,9\% den wichtigen Daten mehr Aufmerksamkeit. Laut Acronis Studie liegen die wichtigen Daten bei über $54 \%$ in Form von Office-Dokumenten (Word, Excel, Powerpoint) und PDFs vor, gefolgt von Fotos (25,4\%). Weit abgeschlagen davon sind beispielsweise Musik (4,3\%), Videos (2,3\%) und Kontakte $(2,2 \%)$. Besonders große Unterschiede gab es hier erneut zwischen den Generationen. Während die Teilnehmer unter 40 Jahren vermehrt Fotos als wichtige Daten nannten (29,8\% bzw. 34,6\%), waren es im Alter ab 50+ hauptsächlich Office-Dokumente $(65,4 \%$ bzw. 59,3\%).

Jeder zweite Deutsche von Datenverlust betroffen Jeder zweite Deutsche hat bereits Daten verloren, bei den Teilnehmern unter 30 Jahren ist die Quote mit 65\% sogar am Höchsten. Besonders davon betroffen waren vor allem Fotos (48\%) und OfficeDokumente (34,5\%). 64,4\% der ältere Generation 60+ gab an, noch nie einen Datenverlust erlebt zu haben. Trotz aller Schwierigkeiten wären insgesamt weniger als $80 \%$ bereit Geld für die Wiederherstellung ihrer Daten zu zahlen, sollten sie diese einmal verlieren. Über 36\% würden nur bis zu 50 Euro investieren.

Die repräsentative Acronis Backup-Studie wurde von der GfK im Zeitraum vom 23. - 28. Januar 2014 in Deutschland durchgeführt.

Weitere Informationen unter: http://www.acronis.de/de-de/ personal/pc-backup/

\section{Europäisches Parlament: elDAS-Verordnung ${ }^{1}$ angenommen}

Auf Grundlage des von Marita Ulvskog vorgetragenen Berichtes über den Vorschlag für eine Verordnung des Europäischen Parlaments und des Rates über die elektronische Identifizierung und Vertrauensdienste für elektronische Transaktionen im Binnenmarkt (Referenz: A7-0365/2013, http://www.europarl.europa.eu/news/de/ news-room/plenary/2014-04-02) vom 06.11.2013 hat das Europäi-

\footnotetext{
1 Siehe auch DuD 2014, Heft4, S.267
} 